\title{
Klein-Beltrami Model. Part I
}

\author{
Roland Coghetto \\ Rue de la Brasserie 5 \\ 7100 La Louvière, Belgium
}

\begin{abstract}
Summary. Tim Makarios (with Isabelle/HOL ${ }^{1}$ ) and John Harrison (with HOL-Light ${ }^{2}$ ) shown that "the Klein-Beltrami model of the hyperbolic plane satisfy all of Tarski's axioms except his Euclidean axiom" 3], 4], 14], 5].

With the Mizar system [2, 7] we use some ideas are taken from Tim Makarios' MSc thesis [13. for the formalization of some definitions (like the absolute) and lemmas necessary for the verification of the independence of the parallel postulate. This work can be also treated as further development of Tarski's geometry in the formal setting [6]. Note that the model presented here, may also be called "Beltrami-Klein Model", "Klein disk model", and the "Cayley-Klein model" 1 .
\end{abstract}

MSC: 51A05 51M10 03B35

Keywords: Tarski's geometry axioms; foundations of geometry; Klein-Beltrami model

MML identifier: BKMODEL1, version: 8.1.07 5.47.1318

\section{Preliminaries}

From now on $a, b, c, d, e, f$ denote real numbers, $g$ denotes a positive real number, $x, y$ denote complex numbers, $S, T$ denote elements of $\mathcal{R}^{2}$, and $u, v$, $w$ denote elements of $\mathcal{E}_{\mathrm{T}}^{3}$.

Now we state the propositions:

(1) Let us consider elements $P_{1}, P_{2}, P_{3}$ of the projective space over $\mathcal{E}_{\mathrm{T}}^{3}$. Suppose $u$ is not zero and $v$ is not zero and $w$ is not zero and $P_{1}=$ the direction of $u$ and $P_{2}=$ the direction of $v$ and $P_{3}=$ the direction of $w$. Then $P_{1}, P_{2}$ and $P_{3}$ are collinear if and only if $\langle|u, v, w|\rangle=0$.

\footnotetext{
1 https://www.isa-afp.org/entries/Tarskis_Geometry.html

2 https://github.com/jrh13/hol-light/blob/master/100/independence.ml 
(2) If $(a \neq 0$ or $b \neq 0)$ and $a \cdot d=b \cdot c$, then there exists $e$ such that $c=e \cdot a$ and $d=e \cdot b$.

(3) If $a^{2}+b^{2}=1$ and $(c \cdot a)^{2}+(c \cdot b)^{2}=1$, then $c=1$ or $c=-1$.

(4) $a \cdot u+(-a) \cdot u=0_{\mathcal{E}_{\mathrm{T}}^{3}}$.

(5) If $0 \leqslant a$ and $c<0$ and $\Delta(a, b, c)=0$, then $a=0$.

Proof: $0 \leqslant b^{2}$.

(6) $\sum\left({ }^{2}(T-S)\right)=\sum\left({ }^{2}(S-T)\right)$.

(7) If $a^{2}+b^{2}=1$ and $c^{2}+d^{2}=1$ and $c \cdot a+d \cdot b=1$, then $b \cdot c=a \cdot d$.

(8) If $a^{2}+b^{2}=1$ and $a=0$, then $b=1$ or $b=-1$.

(9) $0 \leqslant a^{2}$.

(10) If $(a \cdot b)^{2}+b^{2}=1$, then $b=\frac{1}{\sqrt{1+a^{2}}}$ or $b=\frac{-1}{\sqrt{1+a^{2}}}$.

(11) If $a \neq 0$ and $b^{2}=1+a \cdot a$, then $a \cdot \frac{1}{b} \cdot a \cdot \frac{-1}{b}+\frac{1}{b} \cdot \frac{-1}{b}=-1$.

PROOF: $b \neq 0$.

(12) $a^{2} \cdot \frac{1}{b^{2}}=\left(\frac{a}{b}\right)^{2}$.

(13) $a^{2}+b^{2}=1$ if and only if $[a, b] \in \operatorname{circle}(0,0,1)$.

(14) $a^{2}+b^{2}=g^{2}$ if and only if $[a, b] \in \operatorname{circle}(0,0, g)$.

(15) If $a \neq 0$ and $-1<a<1$ and $b=\frac{2+\sqrt{\Delta(a \cdot a,-2,1)}}{2 \cdot a \cdot a}$, then $(1+a \cdot a) \cdot b \cdot b-$ $2 \cdot b+1-b \cdot b=0$.

Proof: $0 \leqslant 1-a^{2} \cdot \Delta(a \cdot a,-2,1) \geqslant 0$.

(16) Suppose $a^{2}+b^{2}=1$ and $-1<c<1$. Then there exists $d$ and there exists $e$ and there exists $f$ such that $e=d \cdot c \cdot a+(1-d) \cdot(-b)$ and $f=d \cdot c \cdot b+(1-d) \cdot a$ and $e^{2}+f^{2}=d^{2}$.

(17) If $a^{2}+b^{2}<1$ and $c^{2}+d^{2}=1$, then $\left(\frac{a+c}{2}\right)^{2}+\left(\frac{b+d}{2}\right)^{2}<1$.

(18) If $|S|^{2} \leqslant 1$, then $0 \leqslant \Delta\left(\sum\left({ }^{2}(T-S)\right), b, \sum\left({ }^{2} S\right)-1\right)$.

(19) If $a^{2}+b^{2}$ is negative, then $a=0$ and $b=0$.

(20) If $u=[a, b, 1]$ and $v=[c, d, 1]$ and $w=\left[\frac{a+c}{2}, \frac{b+d}{2}, 1\right]$, then $\langle|u, v, w|\rangle=0$.

(21) (i) $a \cdot|(u, v)|=|(a \cdot u, v)|$, and

(ii) $a \cdot|(u, v)|=|(u, a \cdot v)|$.

In the sequel $a, b, c$ denote elements of $\mathbb{R}_{\mathrm{F}}$ and $M, N$ denote square matrices over $\mathbb{R}_{\mathrm{F}}$ of dimension 3 .

Now we state the propositions:

(22) If $M=\operatorname{symmetric} 3(0,0,0,0,0,0)$, then $\operatorname{Det} M=0_{\mathbb{R}_{\mathrm{F}}}$.

(23) Suppose $N=\langle\langle a, 0,0\rangle,\langle 0, b, 0\rangle,\langle 0,0, c\rangle\rangle$. Then

(i) $M^{\mathrm{T}} \cdot(N \cdot M)_{1,1}=a \cdot\left(M_{1,1}\right) \cdot\left(M_{1,1}\right)+b \cdot\left(M_{2,1}\right) \cdot\left(M_{2,1}\right)+c \cdot\left(M_{3,1}\right) \cdot\left(M_{3,1}\right)$, and 
(ii) $M^{\mathrm{T}} \cdot(N \cdot M)_{1,2}=a \cdot\left(M_{1,1}\right) \cdot\left(M_{1,2}\right)+b \cdot\left(M_{2,1}\right) \cdot\left(M_{2,2}\right)+c \cdot\left(M_{3,1}\right) \cdot\left(M_{3,2}\right)$, and

(iii) $M^{\mathrm{T}} \cdot(N \cdot M)_{1,3}=a \cdot\left(M_{1,1}\right) \cdot\left(M_{1,3}\right)+b \cdot\left(M_{2,1}\right) \cdot\left(M_{2,3}\right)+c \cdot\left(M_{3,1}\right) \cdot\left(M_{3,3}\right)$, and

(iv) $M^{\mathrm{T}} \cdot(N \cdot M)_{2,1}=a \cdot\left(M_{1,2}\right) \cdot\left(M_{1,1}\right)+b \cdot\left(M_{2,2}\right) \cdot\left(M_{2,1}\right)+c \cdot\left(M_{3,2}\right) \cdot\left(M_{3,1}\right)$, and

(v) $M^{\mathrm{T}} \cdot(N \cdot M)_{2,2}=a \cdot\left(M_{1,2}\right) \cdot\left(M_{1,2}\right)+b \cdot\left(M_{2,2}\right) \cdot\left(M_{2,2}\right)+c \cdot\left(M_{3,2}\right) \cdot\left(M_{3,2}\right)$, and

(vi) $M^{\mathrm{T}} \cdot(N \cdot M)_{2,3}=a \cdot\left(M_{1,2}\right) \cdot\left(M_{1,3}\right)+b \cdot\left(M_{2,2}\right) \cdot\left(M_{2,3}\right)+c \cdot\left(M_{3,2}\right) \cdot\left(M_{3,3}\right)$, and

(vii) $M^{\mathrm{T}} \cdot(N \cdot M)_{3,1}=a \cdot\left(M_{1,3}\right) \cdot\left(M_{1,1}\right)+b \cdot\left(M_{2,3}\right) \cdot\left(M_{2,1}\right)+c \cdot\left(M_{3,3}\right) \cdot\left(M_{3,1}\right)$, and

(viii) $M^{\mathrm{T}} \cdot(N \cdot M)_{3,2}=a \cdot\left(M_{1,3}\right) \cdot\left(M_{1,2}\right)+b \cdot\left(M_{2,3}\right) \cdot\left(M_{2,2}\right)+c \cdot\left(M_{3,3}\right) \cdot\left(M_{3,2}\right)$, and

(ix) $M^{\mathrm{T}} \cdot(N \cdot M)_{3,3}=a \cdot\left(M_{1,3}\right) \cdot\left(M_{1,3}\right)+b \cdot\left(M_{2,3}\right) \cdot\left(M_{2,3}\right)+c \cdot\left(M_{3,3}\right) \cdot\left(M_{3,3}\right)$.

(24) Let us consider natural numbers $m, n$, a square matrix $M$ over $\mathbb{R}_{\mathrm{F}}$ of dimension $m$, and a matrix $N$ over $\mathbb{R}_{\mathrm{F}}$ of dimension $m \times n$. Suppose $m>0$. Then $M \cdot N$ is a matrix over $\mathbb{R}_{\mathrm{F}}$ of dimension $m \times n$.

In the sequel $D$ denotes a non empty set, $d_{1}, d_{2}, d_{3}$ denote elements of $D$, $A$ denotes a matrix over $D$ of dimension $1 \times 3$, and $B$ denotes a matrix over $D$ of dimension $3 \times 1$.

Now we state the propositions:

(25) Let us consider a square matrix $M$ over $D$ of dimension 1 . Then $M^{\mathrm{T}}=$ $M$.

(26) $A^{\mathrm{T}}$ is 3,1 -size.

(27) $\left\langle\left\langle d_{1}, d_{2}, d_{3}\right\rangle\right\rangle$ is a matrix over $D$ of dimension $1 \times 3$.

(28) $\left\langle\left\langle d_{1}\right\rangle,\left\langle d_{2}\right\rangle,\left\langle d_{3}\right\rangle\right\rangle$ is a matrix over $D$ of dimension $3 \times 1$.

(29) $A=\left\langle\left\langle A_{1,1}, A_{1,2}, A_{1,3}\right\rangle\right\rangle$.

Proof: Reconsider $B=\left\langle\left\langle A_{1,1}, A_{1,2}, A_{1,3}\right\rangle\right\rangle$ as a matrix over $D$ of dimension $1 \times 3$. For every natural numbers $i, j$ such that $\langle i, j\rangle \in$ the indices of $A$ holds $A_{i, j}=B_{i, j}$.

(30) $B=\left\langle\left\langle B_{1,1}\right\rangle,\left\langle B_{2,1}\right\rangle,\left\langle B_{3,1}\right\rangle\right\rangle$.

Proof: Reconsider $C=\left\langle\left\langle B_{1,1}\right\rangle,\left\langle B_{2,1}\right\rangle,\left\langle B_{3,1}\right\rangle\right\rangle$ as a matrix over $D$ of dimension $3 \times 1$. For every natural numbers $i, j$ such that $\langle i, j\rangle \in$ the indices of $B$ holds $B_{i, j}=C_{i, j}$.

(31) $\quad A^{\mathrm{T}}=\left\langle\left\langle A_{1,1}\right\rangle,\left\langle A_{1,2}\right\rangle,\left\langle A_{1,3}\right\rangle\right\rangle$. The theorem is a consequence of (26) and (30). 
(32) There exists $d_{1}$ and there exists $d_{2}$ and there exists $d_{3}$ such that $A=\left\langle\left\langle d_{1}\right.\right.$, $\left.\left.d_{2}, d_{3}\right\rangle\right\rangle$. The theorem is a consequence of $(29)$.

(33) Let us consider a finite sequence $p$ of elements of $\mathbb{R}^{1}$. If len $p=3$, then ColVec2Mx $(\mathrm{M} 2 \mathrm{~F}(p))=p$. The theorem is a consequence of $(30)$.

(34) Let us consider a square matrix $M$ over $\mathbb{R}_{\mathrm{F}}$ of dimension 3 , a square matrix $M_{1}$ over $\mathbb{R}$ of dimension 3 , an element $v$ of $\mathcal{E}_{\mathrm{T}}^{3}$, a finite sequence $u_{1}$ of elements of $\mathbb{R}_{\mathrm{F}}$, a finite sequence $u_{2}$ of elements of $\mathbb{R}$, and a finite sequence $p$ of elements of $\mathbb{R}^{1}$. Suppose $p=M \cdot u_{1}$ and $v=\operatorname{M} 2 \mathrm{~F}(p)$ and len $u_{1}=3$ and $u_{1}=u_{2}$ and $M_{1}=M$. Then $v=M_{1} \cdot u_{2}$.

(35) Let us consider a square matrix $N$ over $\mathbb{R}$ of dimension 3 , and a finite sequence $u_{1}$ of elements of $\mathbb{R}$. If $u_{1}=0_{\mathcal{E}_{\mathrm{T}}^{3}}$, then $N \cdot u_{1}=0_{\mathcal{E}_{\mathrm{T}}^{3}}$.

(36) Let us consider a square matrix $N$ over $\mathbb{R}$ of dimension 3 , a finite sequence $u_{1}$ of elements of $\mathbb{R}$, and an element $u$ of $\mathcal{E}_{\mathrm{T}}^{3}$. Suppose $N$ is invertible and $u=u_{1}$ and $u$ is not zero. Then $N \cdot u_{1} \neq 0_{\mathcal{E}_{\mathrm{T}}^{3}}$. The theorem is a consequence of (35).

(37) Let us consider an invertible square matrix $N$ over $\mathbb{R}_{\mathrm{F}}$ of dimension 3 , a square matrix $N_{2}$ over $\mathbb{R}$ of dimension 3 , elements $P, Q$ of the projective space over $\mathcal{E}_{\mathrm{T}}^{3}$, non zero elements $u, v$ of $\mathcal{E}_{\mathrm{T}}^{3}$, and finite sequences $v_{1}, u_{2}$ of elements of $\mathbb{R}$. Suppose $P=$ the direction of $u$ and $Q=$ the direction of $v$ and $u=u_{2}$ and $v=v_{1}$ and $N=N_{2}$ and $N_{2} \cdot u_{2}=v_{1}$. Then (the homography of $N)(P)=Q$. The theorem is a consequence of (34).

(38) Let us consider an invertible square matrix $N$ over $\mathbb{R}_{\mathrm{F}}$ of dimension 3 , a square matrix $N_{2}$ over $\mathbb{R}$ of dimension 3 , elements $P, Q$ of the projective space over $\mathcal{E}_{\mathrm{T}}^{3}$, non zero elements $u, v$ of $\mathcal{E}_{\mathrm{T}}^{3}$, finite sequences $v_{1}, u_{2}$ of elements of $\mathbb{R}$, and a non zero real number $a$. Suppose $P=$ the direction of $u$ and $Q=$ the direction of $v$ and $u=u_{2}$ and $v=v_{1}$ and $N=N_{2}$ and $N_{2} \cdot u_{2}=a \cdot v_{1}$. Then (the homography of $\left.N\right)(P)=Q$. The theorem is a consequence of (34) and (36).

Let us consider a finite sequence $p$ of elements of $\mathbb{R}$ and a square matrix $M$ over $\mathbb{R}$ of dimension 3 . Now we state the propositions:

(39) If len $p=3$, then $|(a \cdot p, M \cdot(b \cdot p))|=a \cdot b \cdot|(p, M \cdot p)|$.

(40) If len $p=3$, then SumAll QuadraticForm $(a \cdot p, M, b \cdot p)=$ $a \cdot b \cdot(\operatorname{SumAll}$ QuadraticForm $(p, M, p))$. The theorem is a consequence of (39).

(41) Let us consider real numbers $a, b$. Then $[a, b, 1]$ is not zero.

(42) Let us consider an element $P$ of $\mathcal{E}_{\mathrm{T}}^{2}$, an element $Q$ of $\mathcal{E}_{\mathrm{T}}^{2}$, and a real number $r$. Then $P \in \operatorname{Sphere}(Q, r)$ if and only if $P \in \operatorname{circle}(Q(1), Q(2), r)$.

In the sequel $u, v$ denote non zero elements of $\mathcal{E}_{\mathrm{T}}^{3}$. 
(43) If the direction of $u=$ the direction of $v$ and $u(3)=v(3)$ and $v(3) \neq 0$, then $u=v$.

The functor Dir101 yielding a point of the projective space over $\mathcal{E}_{\mathrm{T}}^{3}$ is defined by the term

(Def. 1) the direction of $[1,0,1]$.

The functor Dirm101 yielding a point of the projective space over $\mathcal{E}_{\mathrm{T}}^{3}$ is defined by the term

(Def. 2) the direction of $[-1,0,1]$.

The functor Dir011 yielding a point of the projective space over $\mathcal{E}_{\mathrm{T}}^{3}$ is defined by the term

(Def. 3) the direction of $[0,1,1]$.

Now we state the propositions:

(44) (i) Dir101, Dirm101 and Dir011 are not collinear, and

(ii) Dir101, Dirm101 and Dir010 are not collinear, and

(iii) Dir101, Dir011 and Dir010 are not collinear, and

(iv) Dirm101, Dir011 and Dir010 are not collinear.

Proof: Dir101, Dirm101 and Dir011 are not collinear. Dir101, Dirm101 and Dir010 are not collinear. Dir101, Dir011 and Dir010 are not collinear. Dirm101, Dir011 and Dir010 are not collinear.

(45) $\operatorname{symmetric} 3(1,1,1,0,0,0)=I_{\mathbb{R}_{\mathrm{F}}}^{3 \times 3}$.

(46) Let us consider elements $r, a, b, c, d, e, f, g, h, i$ of $\mathbb{R}_{\mathrm{F}}$, and a square matrix $M$ over $\mathbb{R}_{\mathrm{F}}$ of dimension 3. Suppose $M=\langle\langle a, b, c\rangle,\langle d, e, f\rangle,\langle g, h$, $i\rangle\rangle$. Then $r \cdot M=\langle\langle r \cdot a, r \cdot b, r \cdot c\rangle,\langle r \cdot d, r \cdot e, r \cdot f\rangle,\langle r \cdot g, r \cdot h, r \cdot i\rangle\rangle$.

(47) Let us consider a real number $a$, and an element $r$ of $\mathbb{R}_{\mathrm{F}}$. Suppose $r=$ $a \cdot a$. Then $(\operatorname{symmetric} 3(a, a,-a, 0,0,0)) \cdot(\operatorname{symmetric} 3(a, a,-a, 0,0,0))=$ $r \cdot\left(I_{\mathbb{R}_{F}}^{3 \times 3}\right)$. The theorem is a consequence of $(46)$.

Let us consider a non zero real number $a$. Now we state the propositions:

(48) $\quad(\operatorname{symmetric} 3(a, a,-a, 0,0,0)) \cdot\left(\operatorname{symmetric} 3\left(\frac{1}{a}, \frac{1}{a},-\frac{1}{a}, 0,0,0\right)\right)=I_{\mathbb{R}_{\mathrm{F}}}^{3 \times 3}$.

(49) (symmetric $\left.3\left(\frac{1}{a}, \frac{1}{a},-\frac{1}{a}, 0,0,0\right)\right) \cdot(\operatorname{symmetric} 3(a, a,-a, 0,0,0))=I_{\mathbb{R}_{\mathrm{F}}}^{3 \times 3}$. The theorem is a consequence of (48).

(50) $(\operatorname{symmetric} 3(1,1,-1,0,0,0)) \cdot(\operatorname{symmetric} 3(1,1,-1,0,0,0))=I_{\mathbb{R}_{\mathrm{F}}}^{3 \times 3}$. The theorem is a consequence of (48).

(51) Let us consider a non zero real number $a$, and a square matrix $N$ over $\mathbb{R}_{\mathrm{F}}$ of dimension 3 . If $N=\operatorname{symmetric} 3(a, a,-a, 0,0,0)$, then $N$ is invertible. The theorem is a consequence of (48) and (49).

(52) (i) symmetric3 $(1,1,-1,0,0,0)$ is an invertible square matrix over $\mathbb{R}_{F}$ of dimension 3 , and 
(ii) $(\operatorname{symmetric} 3(1,1,-1,0,0,0))^{\smile}=\operatorname{symmetric} 3(1,1,-1,0,0,0)$. The theorem is a consequence of (50).

(53) Let us consider an invertible square matrix $N$ over $\mathbb{R}_{\mathrm{F}}$ of dimension 3 , a square matrix $N_{1}$ over $\mathbb{R}_{\mathrm{F}}$ of dimension 3 , and square matrices $M$, $N_{2}$ over $\mathbb{R}$ of dimension 3 . Suppose $M=\operatorname{symmetric} 3(1,1,-1,0,0,0)$ and $N_{1}=M$ and $N_{2}=\left(\mathbb{R}_{\mathrm{F}} \rightarrow \mathbb{R}\right) N^{\smile}$. Then $N^{\mathrm{T}} \cdot N_{1} \cdot N=\left(\left(\mathbb{R}_{\mathrm{F}} \rightarrow \mathbb{R}\right)((\mathbb{R} \rightarrow\right.$ $\left.\left.\left.\mathbb{R}_{\mathrm{F}}\right) N_{2}^{\mathrm{T}}\right)^{\smile}\right) \cdot M \cdot\left(\left(\mathbb{R}_{\mathrm{F}} \rightarrow \mathbb{R}\right)\left(\left(\mathbb{R} \rightarrow \mathbb{R}_{\mathrm{F}}\right) N_{2}\right)^{\smile}\right)$.

Proof: $\left(\mathbb{R}_{\mathrm{F}} \rightarrow \mathbb{R}\right)\left(\left(\mathbb{R} \rightarrow \mathbb{R}_{\mathrm{F}}\right) N_{2}^{\mathrm{T}}\right)^{\smile}=N^{\mathrm{T}}$ by [15, (13), (16)].

(54) Let us consider a natural number $n$, an element $a$ of $\mathbb{R}_{\mathrm{F}}$, a real number $r$, a square matrix $A$ over $\mathbb{R}_{\mathrm{F}}$ of dimension $n$, and a square matrix $r_{1}$ over $\mathbb{R}$ of dimension $n$. If $a=r$ and $A=r_{1}$, then $a \cdot A=r \cdot r_{1}$.

(55) Let us consider a natural number $n$, an element $a$ of $\mathbb{R}_{\mathrm{F}}$, and square matrices $A, B$ over $\mathbb{R}_{\mathrm{F}}$ of dimension $n$. If $n>0$, then $(a \cdot A) \cdot B=a \cdot(A \cdot B)$. The theorem is a consequence of (54).

(56) $\operatorname{symmetric} 3(a, a,-a, 0,0,0)=a \cdot(\operatorname{symmetric} 3(1,1,-1,0,0,0))$. The theorem is a consequence of $(46)$.

(57) If $M=\operatorname{symmetric} 3(a, a,-a, 0,0,0)$, then $M \cdot M \cdot M=$ $a \cdot a \cdot a \cdot($ symmetric $3(1,1,-1,0,0,0))$. The theorem is a consequence of (47), (55), and (56).

Let us consider a natural number $n$, a real number $a$, a square matrix $M$ over $\mathbb{R}$ of dimension $n$, and a finite sequence $x$ of elements of $\mathbb{R}$. Now we state the propositions:

(58) If $n>0$ and len $x=n$, then $M \cdot(a \cdot x)=(a \cdot M) \cdot x$.

(59) If $n>0$ and len $x=n$, then $a \cdot(M \cdot x)=(a \cdot M) \cdot x$. The theorem is a consequence of (58).

(60) Let us consider a natural number $n$, and a square matrix $N$ over $\mathbb{R}$ of dimension $n$. Suppose $N$ is invertible. Then

(i) $N^{\mathrm{T}}$ is invertible, and

(ii) $\operatorname{Inv} N^{\mathrm{T}}=(\operatorname{Inv} N)^{\mathrm{T}}$.

(61) Let us consider a non zero real number $r$, and matrices $N, O, M$ over $\mathbb{R}$ of dimension $3 \times 3$. Suppose $N$ is invertible and $M=r \cdot O$ and $M=N^{\mathrm{T}} \cdot O \cdot N$. Then $(\operatorname{Inv} N)^{\mathrm{T}} \cdot O \cdot(\operatorname{Inv} N)=\frac{1}{r} \cdot O$. The theorem is a consequence of $(60)$.

(62) Let us consider a real number $r$, square matrices $M, N$ over $\mathbb{R}_{\mathrm{F}}$ of dimension 3, and square matrices $M_{1}, N_{2}$ over $\mathbb{R}$ of dimension 3. Suppose $M_{1}=M$ and $N_{2}=N$ and $N$ is symmetric and $M_{1}=r \cdot N_{2}$. Then $M$ is symmetric.

Let us consider a real number $r$ and square matrices $O, M$ over $\mathbb{R}$ of dimension 3. Now we state the propositions: 
(63) Suppose $O=\operatorname{symmetric} 3(1,1,-1,0,0,0)$ and $M=r \cdot O$. Then

(i) $O \cdot M=r \cdot\left(1_{\mathbb{R}} \operatorname{matrix}(3)\right)$, and

(ii) $M \cdot O=r \cdot\left(1_{\mathbb{R}} \operatorname{matrix}(3)\right)$.

The theorem is a consequence of (50).

(64) If $O=\operatorname{symmetric} 3(1,1,-1,0,0,0)$ and $M=r \cdot O$, then $\left(M^{\mathrm{T}} \cdot O\right)^{\mathrm{T}} \cdot O$. $\left(M^{\mathrm{T}} \cdot O\right)=r^{2} \cdot O$.

PROOF: Reconsider $M_{1}=M$ as a square matrix over $\mathbb{R}_{\mathrm{F}}$ of dimension 3 . $M_{1}$ is symmetric. $r \cdot\left(1_{\mathbb{R}} \operatorname{matrix}(3)\right) \cdot O \cdot\left(r \cdot\left(1_{\mathbb{R}} \operatorname{matrix}(3)\right)\right)=r^{2} \cdot O$.

(65) Let us consider square matrices $O, N$ over $\mathbb{R}$ of dimension 3 . Then $\left(N^{\mathrm{T}}\right.$. $O)^{\mathrm{T}} \cdot O \cdot\left(N^{\mathrm{T}} \cdot O\right)=\left(O^{\mathrm{T}} \cdot\left(N \cdot O \cdot N^{\mathrm{T}}\right)\right) \cdot O$.

(66) Let us consider square matrices $N_{2}, M_{1}$ over $\mathbb{R}$ of dimension 3 , and finite sequences $p_{1}, p_{2}, p_{3}$ of elements of $\mathbb{R}$. Suppose $p_{1}=\langle 1,0,0\rangle$ and $p_{2}=\langle 0$, $1,0\rangle$ and $p_{3}=\langle 0,0,1\rangle$ and $N_{2} \cdot p_{1}=M_{1} \cdot p_{1}$ and $N_{2} \cdot p_{2}=M_{1} \cdot p_{2}$ and $N_{2} \cdot p_{3}=M_{1} \cdot p_{3}$. Then $N_{2}=M_{1}$.

(67) Let us consider a non zero real number $a$, and an element $u$ of $\mathcal{E}_{\mathrm{T}}^{3}$. If $a \cdot u=0_{\mathcal{E}_{\mathrm{T}}^{3}}$, then $u$ is zero.

(68) Let us consider non zero elements $u, v$ of $\mathcal{E}_{\mathrm{T}}^{3}$, and real numbers $a, b$. Suppose $(a \neq 0$ or $b \neq 0)$ and $a \cdot u+b \cdot v=0_{\mathcal{E}_{\mathrm{T}}^{3}}$. Then $u$ and $v$ are proportional.

Proof: Reconsider $a_{1}=a \cdot u, b_{1}=b \cdot v$ as an element of $\mathcal{E}_{\mathrm{T}}^{3}$. Consider $c$ being a real number such that $c \neq 0$ and $a_{1}=c \cdot b_{1} . a \neq 0$ and $b \neq 0$ by [11, (3), (1)].

(69) Let us consider an invertible square matrix $N$ over $\mathbb{R}_{\mathrm{F}}$ of dimension 3 , and points $P, Q, R$ of the projective space over $\mathcal{E}_{\mathrm{T}}^{3}$. Suppose $P \neq Q$ and (the homography of $N)(P)=Q$ and (the homography of $N)(Q)=P$ and $P, Q$ and $R$ are collinear. Then (the homography of $N)(($ the homography of $N)(R))=R$.

Proof: Consider $u_{1}, v_{1}$ being elements of $\mathcal{E}_{\mathrm{T}}^{3}, u_{4}$ being a finite sequence of elements of $\mathbb{R}_{\mathrm{F}}, p_{1}$ being a finite sequence of elements of $\mathbb{R}^{1}$ such that $P=$ the direction of $u_{1}$ and $u_{1}$ is not zero and $u_{1}=u_{4}$ and $p_{1}=N \cdot u_{4}$ and $v_{1}=\operatorname{M} 2 \mathrm{~F}\left(p_{1}\right)$ and $v_{1}$ is not zero and (the homography of $\left.N\right)(P)=$ the direction of $v_{1}$. Consider $u_{2}, v_{2}$ being elements of $\mathcal{E}_{\mathrm{T}}^{3}, u_{5}$ being a finite sequence of elements of $\mathbb{R}_{\mathrm{F}}, p_{2}$ being a finite sequence of elements of $\mathbb{R}^{1}$ such that $Q=$ the direction of $u_{2}$ and $u_{2}$ is not zero and $u_{2}=u_{5}$ and $p_{2}=N \cdot u_{5}$ and $v_{2}=\mathrm{M} 2 \mathrm{~F}\left(p_{2}\right)$ and $v_{2}$ is not zero and (the homography of $N)(Q)=$ the direction of $v_{2}$. Consider $u_{3}$ being an element of $\mathcal{E}_{\mathrm{T}}^{3}$ such that $u_{3}$ is not zero and $R=$ the direction of $u_{3}$. Consider $l_{1}$ being a real number such that $l_{1} \neq 0$ and $v_{2}=l_{1} \cdot u_{1}$. Consider $l_{2}$ being a real number 
such that $l_{2} \neq 0$ and $v_{1}=l_{2} \cdot u_{2} \cdot\left\langle\left|u_{1}, u_{2}, u_{3}\right|\right\rangle=0$. Consider $a, b, c$ being real numbers such that $a \cdot u_{1}+b \cdot u_{2}+c \cdot u_{3}=0_{\mathcal{E}_{T}^{3}}$ and $(a \neq 0$ or $b \neq 0$ or $c \neq 0) . c \neq 0$. (The homography of $N \cdot N)(R)=\stackrel{R}{R}$.

(70) Let us consider a natural number $n$, elements $u, v$ of $\mathcal{E}_{\mathrm{T}}^{n}$, and real numbers $a, b$. If $(1-a) \cdot u+a \cdot v=(1-b) \cdot v+b \cdot u$, then $(1-(a+b)) \cdot u=(1-(a+b)) \cdot v$. Proof: Reconsider $r_{1}=u, r_{2}=v$ as an element of $\mathcal{R}^{n}$. $(1-a) \cdot r_{1}+a$. $r_{2}-a \cdot r_{2}=(1-a) \cdot r_{1} \cdot(1-b) \cdot r_{2}-a \cdot r_{2}+b \cdot r_{1}-b \cdot r_{1}=(1-b) \cdot r_{2}-a \cdot r_{2}$.

(71) The projective space over $\mathcal{E}_{\mathrm{T}}^{3}$ is proper.

The real projective plane yielding a non empty, proper projective plane defined in terms of collinearity is defined by the term

(Def. 4) the projective space over $\mathcal{E}_{\mathrm{T}}^{3}$.

From now on $P, Q, R$ denote points of Inc-ProjSp(the real projective plane), $L$ denotes a line of Inc-ProjSp(the real projective plane), and $p, q, r$ denote points of the real projective plane.

Now we state the propositions:

(72) Let us consider an element $L$ of $L$ (the real projective plane). Then there exists $p$ and there exists $q$ such that $p \neq q$ and $L=\operatorname{Line}(p, q)$.

(73) There exists $p$ and there exists $q$ such that $p \neq q$ and $L=\operatorname{Line}(p, q)$.

(74) If $R=r$ and $L=\operatorname{Line}(p, q)$, then $R$ lies on $L$ iff $p, q$ and $r$ are collinear.

(75) Inc-ProjSp(the real projective plane) is an incidence projective plane. Proof: Inc-ProjSp(the real projective plane) is 2-dimensional.

(76) Let us consider lines $L_{1}, L_{2}$ of the real projective plane. Then $L_{1}$ meets $L_{2}$. The theorem is a consequence of (75).

In the sequel $u, v, w$ denote non zero elements of $\mathcal{E}_{\mathrm{T}}^{3}$.

(77) Suppose $p=$ the direction of $u$ and $q=$ the direction of $v$ and $R=$ the direction of $w$ and $L=\operatorname{Line}(p, q)$. Then $R$ lies on $L$ if and only if $\langle|u, v, w|\rangle=0$. The theorem is a consequence of (74).

(78) Let us consider elements $p, q$ of the projective space over $\mathcal{E}_{\mathrm{T}}^{3}$. Suppose $p \neq q$ and $p=$ the direction of $u$ and $q=$ the direction of $v$. Then $u \times v$ is not zero.

Let $p, q$ be points of the real projective plane. Assume $p \neq q$. The functor $\mathrm{L} 2 \mathrm{P}(p, q)$ yielding a point of the real projective plane is defined by

(Def. 5) there exist non zero elements $u, v$ of $\mathcal{E}_{\mathrm{T}}^{3}$ such that $p=$ the direction of $u$ and $q=$ the direction of $v$ and $i t=$ the direction of $u \times v$.

Now we state the propositions:

(79) Let us consider points $p, q$ of the real projective plane. Suppose $p \neq q$. Then 
(i) $\mathrm{L} 2 \mathrm{P}(q, p)=\mathrm{L} 2 \mathrm{P}(p, q)$, and

(ii) $p \neq \mathrm{L} 2 \mathrm{P}(p, q)$.

Proof: Consider $u_{1}, v_{1}$ being non zero elements of $\mathcal{E}_{\mathrm{T}}^{3}$ such that $p=$ the direction of $u_{1}$ and $q=$ the direction of $v_{1}$ and $\mathrm{L} 2 \mathrm{P}(p, q)=$ the direction of $u_{1} \times v_{1}$. Consider $u_{2}, v_{2}$ being non zero elements of $\mathcal{E}_{\mathrm{T}}^{3}$ such that $q=$ the direction of $u_{2}$ and $p=$ the direction of $v_{2}$ and $\operatorname{L} 2 \mathrm{P}(q, p)=$ the direction of $u_{2} \times v_{2}$. Consider $a$ being a real number such that $a \neq 0$ and $u_{1}=a \cdot v_{2}$. Consider $b$ being a real number such that $b \neq 0$ and $v_{1}=b \cdot u_{2} \cdot a \cdot v_{2} \times b \cdot u_{2}=(-a \cdot b) \cdot\left(u_{2} \times v_{2}\right) \cdot u_{1} \times v_{1}$ is not zero. $u_{2} \times v_{2}$ is not zero. $p \neq \mathrm{L} 2 \mathrm{P}(p, q)$.

(80) Let us consider an invertible square matrix $N$ over $\mathbb{R}_{\mathrm{F}}$ of dimension 3. Then $\operatorname{dom}\left(\right.$ the homography of $N$ ) $=$ the projective points over $\mathcal{E}_{\mathrm{T}}^{3}$.

\section{Absolute}

Let $a, b, c, d, e, f$ be real numbers. The interior of the conic for $a, b, c, d, e$ and $f$ yielding a subset of the projective space over $\mathcal{E}_{\mathrm{T}}^{3}$ is defined by the term

(Def. 6) $\left\{P\right.$, where $P$ is a point of the projective space over $\mathcal{E}_{\mathrm{T}}^{3}$ : for every element $u$ of $\mathcal{E}_{\mathrm{T}}^{3}$ such that $u$ is not zero and $P=$ the direction of $u$ holds qfconic $(a, b, c, d, e, f, u)$ is negative .

Now we state the proposition:

(81) Let us consider real numbers $a, b, c, d, e, f$, and non zero elements $u_{1}, u_{2}$ of $\mathcal{E}_{\mathrm{T}}^{3}$. Suppose the direction of $u_{1}=$ the direction of $u_{2}$ and qfconic $(a, b, c, d$, $\left.e, f, u_{1}\right)$ is negative. Then $\operatorname{qfconic}\left(a, b, c, d, e, f, u_{2}\right)$ is negative.

The absolute yielding a non empty subset of the projective space over $\mathcal{E}_{\mathrm{T}}^{3}$ is defined by the term

(Def. 7) $\operatorname{conic}(1,1,-1,0,0,0)$.

Now we state the proposition:

(82) Let us consider a square matrix $O$ over $\mathbb{R}$ of dimension 3 , an element $P$ of the projective space over $\mathcal{E}_{\mathrm{T}}^{3}$, and a finite sequence $p$ of elements of $\mathbb{R}$. Suppose $O=$ symmetric $3(1,1,-1,0,0,0)$ and $P=$ the direction of $u$ and $u=$ $p$. Then $P \in$ the absolute if and only if $\operatorname{SumAll}$ QuadraticForm $(p, O, p)=$ 0 . The theorem is a consequence of (40).

Let us consider an element $P$ of the absolute. Now we state the propositions:

(83) If $P=$ the direction of $u$, then $u(3) \neq 0$.

Proof: Consider $Q$ being a point of the projective space over $\mathcal{E}_{\mathrm{T}}^{3}$ such that $P=Q$ and for every element $u$ of $\mathcal{E}_{\mathrm{T}}^{3}$ such that $u$ is not zero and 
$Q=$ the direction of $u$ holds qfconic $(1,1,-1,0,0,0, u)=0 . u(3) \neq 0$ by [8, (3), (4)].

(84) If $P=$ the direction of $u$ and $u(3)=1$, then $[u(1), u(2)] \in \operatorname{circle}(0,0,1)$. The theorem is a consequence of (13).

(85) Let us consider a point $P$ of the projective space over $\mathcal{E}_{\mathrm{T}}^{3}$. Suppose $P=$ the direction of $u$ and $u(3)=1$ and $[u(1), u(2)] \in \operatorname{circle}(0,0,1)$. Then $P$ is an element of the absolute.

(86) Let us consider a point $P$ of the projective space over $\mathcal{E}_{\mathrm{T}}^{3}$, and a non zero element $u$ of $\mathcal{E}_{\mathrm{T}}^{3}$. Suppose $P=$ the direction of $u$ and $u(3)=1$. Then $[u(1), u(2)] \in \operatorname{circle}(0,0,1)$ if and only if $P$ is an element of the absolute.

Let $P$ be an element of the absolute. The absolute to unit circle of $P$ yielding an element of circle $(0,0,1)$ is defined by

(Def. 8) there exists a non zero element $u$ of $\mathcal{E}_{\mathrm{T}}^{3}$ such that $P=$ the direction of $u$ and $u(3)=1$ and $i t=[u(1), u(2)]$.

Now we state the proposition:

(87) The carrier of TopUnitCircle $2=\operatorname{circle}(0,0,1)$.

Proof: The carrier of TopUnitCircle $2 \subseteq \operatorname{circle}(0,0,1)$. circle $(0,0,1) \subseteq$ the carrier of TopUnitCircle 2 by [9, (52)], [10, (9)].

Let $u$ be a non zero element of $\mathcal{E}_{\mathrm{T}}^{2}$. Assume $u \in \operatorname{circle}(0,0,1)$. The unit circle to absolute of $u$ yielding an element of the absolute is defined by the term

(Def. 9) the direction of $[u(1), u(2), 1]$.

Now we state the proposition:

(88) Let us consider an element $u$ of $\mathcal{E}_{\mathrm{T}}^{3}$. Suppose qfconic $(1,1,-1,0,0,0, u)=$ 0 and $u(3)=1$. Then $[u(1), u(2)] \in \operatorname{Sphere}\left(0_{\mathcal{E}_{\mathrm{T}}^{2}}, 1\right)$. The theorem is a consequence of (13).

Let us consider an element $P$ of the absolute. Now we state the propositions:

(89) There exists $u$ such that

(i) $u(1)^{2}+u(2)^{2}=1$, and

(ii) $u(3)=1$, and

(iii) $P=$ the direction of $u$.

The theorem is a consequence of (83), (84), and (14).

(90) There exists an element $Q$ of the absolute such that $P \neq Q$.

Proof: Consider $Q$ being a point of the projective space over $\mathcal{E}_{\mathrm{T}}^{3}$ such that $P=Q$ and for every element $u$ of $\mathcal{E}_{\mathrm{T}}^{3}$ such that $u$ is not zero and $Q=$ the direction of $u$ holds qfconic $(1,1,-1,0,0,0, u)=0$. Consider $u$ being an element of $\mathcal{E}_{\mathrm{T}}^{3}$ such that $u$ is not zero and the direction of $u=P . u(3) \neq$ 0 . $[u(1), u(2),-u(3)]$ is not zero. Reconsider $v=[u(1), u(2),-u(3)]$ as 
a non zero element of $\mathcal{E}_{\mathrm{T}}^{3}$. Reconsider $R=$ the direction of $v$ as an element of the projective space over $\mathcal{E}_{\mathrm{T}}^{3}$. $R \neq P$. For every element $w$ of $\mathcal{E}_{\mathrm{T}}^{3}$ such that $w$ is not zero and $R=$ the direction of $w$ holds qfconic $(1,1,-1,0,0,0, w)=$ 0 .

(91) Let us consider real numbers $a, b$. Suppose $a^{2}+b^{2}=1$. Then $[-b, a, 0]$ is not zero.

(92) Let us consider elements $P, Q, R$ of the absolute. If $P, Q, R$ are mutually different, then $P, Q$ and $R$ are not collinear.

Proof: Consider $u_{12}$ being an element of $\mathcal{E}_{\mathrm{T}}^{3}$ such that $u_{12}$ is not zero and $P=$ the direction of $u_{12}$. Consider $u_{16}$ being an element of $\mathcal{E}_{\mathrm{T}}^{3}$ such that $u_{16}$ is not zero and $Q=$ the direction of $u_{16}$. Consider $u_{20}$ being an element of $\mathcal{E}_{\mathrm{T}}^{3}$ such that $u_{20}$ is not zero and $R=$ the direction of $u_{20}$. Reconsider $u_{13}=\left(u_{12}\right)_{\mathbf{1}}, u_{14}=\left(u_{12}\right)_{\mathbf{2}}, u_{15}=\left(u_{12}\right)_{\mathbf{3}}, u_{17}=\left(u_{16}\right)_{\mathbf{1}}, u_{18}=\left(u_{16}\right)_{\mathbf{2}}$, $u_{19}=\left(u_{16}\right)_{\mathbf{3}}, u_{21}=\left(u_{20}\right)_{\mathbf{1}}, u_{22}=\left(u_{20}\right)_{\mathbf{2}}, u_{23}=\left(u_{20}\right)_{\mathbf{3}}$ as a real number. $u_{12}(3) \neq 0$ and $u_{16}(3) \neq 0$ and $u_{20}(3) \neq 0$. Reconsider $v_{5}=\frac{u_{13}}{u_{15}}, v_{6}=\frac{u_{14}}{u_{15}}$, $v_{8}=\frac{u_{17}}{u_{19}}, v_{9}=\frac{u_{18}}{u_{19}}, v_{11}=\frac{u_{21}}{u_{23}}, v_{12}=\frac{u_{22}}{u_{23}}$ as a real number. Reconsider $v_{4}=$ $\left[v_{5}, v_{6}, 1\right], v_{7}=\left[v_{8}, v_{9}, 1\right], v_{10}=\left[v_{11}, v_{12}, 1\right]$ as a non zero element of $\mathcal{E}_{\mathrm{T}}^{3}$. $P=$ the direction of $v_{4}$ and $Q=$ the direction of $v_{7}$ and $R=$ the direction of $v_{10}$. Consider $t_{1}, t_{2}, t_{3}$ being elements of $\mathcal{E}_{\mathrm{T}}^{3}$ such that $P=$ the direction of $t_{1}$ and $Q=$ the direction of $t_{2}$ and $R=$ the direction of $t_{3}$ and $t_{1}$ is not zero and $t_{2}$ is not zero and $t_{3}$ is not zero and there exist real numbers $a_{1}$, $b_{1}, c_{1}$ such that $a_{1} \cdot t_{1}+b_{1} \cdot t_{2}+c_{1} \cdot t_{3}=0_{\mathcal{E}_{\mathrm{T}}^{3}}$ and $\left(a_{1} \neq 0\right.$ or $b_{1} \neq 0$ or $\left.c_{1} \neq 0\right)$. Consider $a_{1}, b_{1}, c_{1}$ being real numbers such that $a_{1} \cdot t_{1}+b_{1} \cdot t_{2}+c_{1} \cdot t_{3}=0_{\mathcal{E}_{\mathrm{T}}^{3}}$ and $a_{1} \neq 0$ or $b_{1} \neq 0$ or $c_{1} \neq 0$. Consider $l_{1}$ being a real number such that $l_{1} \neq 0$ and $t_{1}=l_{1} \cdot v_{4}$. Consider $l_{2}$ being a real number such that $l_{2} \neq 0$ and $t_{2}=l_{2} \cdot v_{7}$. Consider $l_{3}$ being a real number such that $l_{3} \neq 0$ and $t_{3}=l_{3} \cdot v_{10}$. Reconsider $A=\left[\left(v_{4}\right)_{\mathbf{1}},\left(v_{4}\right)_{\mathbf{2}}\right], B=\left[\left(v_{7}\right)_{\mathbf{1}},\left(v_{7}\right)_{\mathbf{2}}\right], C=\left[\left(v_{10}\right)_{\mathbf{1}}\right.$, $\left.\left(v_{10}\right)_{2}\right]$ as an element of $\mathcal{E}_{\mathrm{T}}^{2} . A \neq B . A \neq C . B \neq C . A \in \operatorname{Sphere}\left(0_{\mathcal{E}_{\mathrm{T}}^{2}}, 1\right)$. qfconic $\left(1,1,-1,0,0,0, v_{7}\right)=0 . B \in \operatorname{Sphere}\left(0_{\mathcal{E}_{\mathrm{T}}^{2}}, 1\right) . C \in \operatorname{Sphere}\left(0_{\mathcal{E}_{\mathrm{T}}^{2}}, 1\right)$.

(93) Let us consider a non zero real number $r$, and invertible square matrices $O, N, M$ over $\mathbb{R}_{\mathrm{F}}$ of dimension 3 . Suppose $O=$ symmetric $3(1,1,-1,0,0,0)$ and $M=\operatorname{symmetric} 3(r, r,-r, 0,0,0)$ and

$M=N^{\mathrm{T}} \cdot O \cdot N$ and (the homography of $\left.M\right)^{\circ}($ the absolute $)=$ the absolute. Then (the homography of $N)^{\circ}$ (the absolute) $=$ the absolute.

Proof: (The homography of $N)^{\circ}$ (the absolute) $\subseteq$ the absolute.

The absolute $\subseteq$ (the homography of $N)^{\circ}$ (the absolute) by [12, (50)]. 


\section{REFERENCES}

[1] Norbert A'Campo and Athanase Papadopoulos. On Klein's so-called non-Euclidean geometry, arXiv preprint arXiv:1406.7309, 2014.

[2] Grzegorz Bancerek, Czesław Byliński, Adam Grabowski, Artur Korniłowicz, Roman Matuszewski, Adam Naumowicz, Karol Pąk, and Josef Urban. Mizar: State-of-the-art and beyond In Manfred Kerber, Jacques Carette, Cezary Kaliszyk, Florian Rabe, and Volker Sorge, editors, Intelligent Computer Mathematics, volume 9150 of Lecture Notes in Computer Science, pages 261-279. Springer International Publishing, 2015. ISBN 978-3319-20614-1. doi $10.1007 / 978-3-319-20615-8 \_17$.

[3] Eugenio Beltrami. Saggio di interpetrazione della geometria non-euclidea. Giornale di Matematiche, 6:284-322, 1868.

[4] Eugenio Beltrami. Essai d'interprétation de la géométrie non-euclidéenne. In Annales scientifiques de l'École Normale Supérieure. Trad. par J. Hoüel, volume 6, pages 251288. Elsevier, 1869.

[5] Karol Borsuk and Wanda Szmielew. Podstawy geometrii. Państwowe Wydawnictwo Naukowe, Warszawa, 1955 (in Polish).

[6] Adam Grabowski. Tarski's geometry modelled in Mizar computerized proof assistant. In Proceedings of the 2016 Federated Conference on Computer Science and Information Systems, FedCSIS 2016, Gdańsk, Poland, September 11-14, 2016, pages 373-381, 2016. doi:10.15439/2016F290.

[7] Adam Grabowski, Artur Korniłowicz, and Adam Naumowicz. Four decades of Mizar. Journal of Automated Reasoning, 55(3):191-198, 2015. doi:10.1007/s10817-015-9345-1

[8] Kanchun, Hiroshi Yamazaki, and Yatsuka Nakamura. Cross products and tripple vector products in 3-dimensional Euclidean space. Formalized Mathematıcs, 11(4):381-383, 2003.

[9] Artur Korniłowicz and Yasunari Shidama. Intersections of intervals and balls in $\mathcal{E}_{\mathrm{T}}^{n}$. Formalized Mathematics, 12(3):301-306, 2004.

[10] Artur Korniłowicz and Yasunari Shidama. Some properties of circles on the plane. Formalized Mathematics, 13(1):117-124, 2005.

[11] Akihiro Kubo. Lines in n-dimensional Euclidean spaces. Formalized Mathematics, 11(4): 371-376, 2003.

[12] Xiquan Liang, Piqing Zhao, and Ou Bai. Vector functions and their differentiation formulas in 3-dimensional Euclidean spaces. Formalized Mathematics, 18(1):1-10, 2010. doi: $10.2478 /$ v10037-010-0001-2

[13] Timothy James McKenzie Makarios. A mechanical verification of the independence of Tarski's Euclidean Axiom. Victoria University of Wellington, New Zealand, 2012. Master's thesis.

[14] Andrzej Trybulec. A Borsuk theorem on homotopy types. Formalized Mathematics, 2(4): 535-545, 1991.

[15] Xiaopeng Yue, Xiquan Liang, and Zhongpin Sun. Some properties of some special matrices Formalized Mathematics, 13(4):541-547, 2005. 